\title{
gु \\ Flow and clogging of a sheep herd passing through a bottleneck
}

\author{
A. Garcimartín, ${ }^{1, *}$ J. M. Pastor, ${ }^{1}$ L. M. Ferrer, ${ }^{2}$ J. J. Ramos, ${ }^{2}$ C. Martín-Gómez, ${ }^{3}$ and I. Zuriguel ${ }^{1}$ \\ ${ }^{1}$ Depto. de Física y Mat. Apl., Fac. Ciencias, Universidad de Navarra, 31080 Pamplona, Spain \\ ${ }^{2}$ Dpto. de Patología Animal, Fac. Veterinaria, Universidad de Zaragoza, c/ Miguel Servet 177, 50013 Zaragoza, Spain \\ ${ }^{3}$ Sección de Instalaciones y Energía, Escuela de Arquitectura, Universidad de Navarra, 31080 Pamplona, Spain
}

(Received 24 December 2014; published 17 February 2015)

\begin{abstract}
We present an experimental study of a flock passing through a narrow door. Video monitoring of daily routines in a farm has enabled us to collect a sizable amount of data. By measuring the time lapse between the passage of consecutive animals, some features of the flow regime can be assessed. A quantitative definition of clogging is demonstrated based on the passage time statistics. These display broad tails, which can be fitted by power laws with a relatively large exponent. On the other hand, the distribution of burst sizes robustly evidences exponential behavior. Finally, borrowing concepts from granular physics and statistical mechanics, we evaluate the effect of increasing the door size and the performance of an obstacle placed in front of it. The success of these techniques opens new possibilities regarding their eventual extension to the management of human crowds.
\end{abstract}

DOI: 10.1103/PhysRevE.91.022808

PACS number(s): 89.40.-a, 45.70.Vn, 87.19.ru

\section{INTRODUCTION}

Collective behavior of human crowds has attracted the attention of physicists in recent years, because it has been shown that the dynamics of people can be represented, at least in some situations, by simple models [1,2]. New approaches have allowed us to consider the dynamics of large amounts of people from a vantage point, with the aim of avoiding or alleviating dangerous situations such as clogging and stampedes of panicking persons. It is evident that the potential benefits that can be derived from these studies are particularly valuable. But these efforts are hindered by the plain difficulty of gathering data against which theories and models can be checked. Three sources can be pointed out. First, data from actual catastrophes have been analyzed [3]. The problem with these data is, of course, that they are collected in a serendipitous way and cannot be rehearsed at will. Second, controlled evacuation drills, as reported for instance in Refs. [4,5], can be performed. But these experiments are not carried out in a competitive situation because of obvious ethics considerations. And, third, computer simulations [6-9] are inspiring and useful, but the rules embodied in the underlying models are difficult to test with controlled experiments, thus limiting their value.

In this framework, studies concerning the motion of large numbers of living beings (see, for instance, Refs. [4,10-12]) are extremely interesting. It must be pointed out that animals move routinely in a much more violent fashion than people use to do, and the dynamics of flocks and herds are relatively easy to observe. Apart from its own interest, the motion of active matter (i.e., self-propelled particles, of which animals are a particular case) can provide an intermediate step between inert particles and humans. Indeed, some ideas used to describe the dynamics of active matter have been borrowed from the fields of granular matter and colloidal suspensions. Carrying out experiments with inert grains in the laboratory is certainly easier than gathering data from a panicking crowd, and animals provide a convenient intermediate step. Nevertheless, as far as we know, only experiments with mice [13] and ants [14,15]

\footnotetext{
*angel@unav.es
}

have been performed. Apart from those, experiments on woodlice have been recently reported by Sobhani et al. [16]. Ants tend to follow one another [17] and are not a good model for humans [15]. Many mammals (such as dogs or horses) do have a leader; others, such as cats, do not flock.

In this context, we have resorted to sheep. In most of the experiments and observations carried out under controlled conditions, persons are instructed not to shove one another. Sheep, on the other hand, push the others vigorously. They are used to competitively hack their way through a dense gathering without getting hurt, and so these situations are not so dangerous for them as they would be for humans. The use of this animal was suggested to be highly useful for testing pedestrian egress conditions [18]. Sheep have many advantages: an all-female herd does not have a leader; sheep are gregarious; their size is in the same order of magnitude than humans; and their running and walking velocity are close to that of a person. In this framework, the aim of this paper is to study whether some concepts that have been demonstrated in granular matter are applicable to the case of active matter, in particular, to sheep.

Bottlenecks are a main concern because a clog can easily form and lead to casualties, if people are involved [19], or to a flow arrest, in the case of granular matter [20-24]. In a recent work [25], strong analogies have been identified in the flow through a constriction of a pedestrian model, sheep, colloidal suspensions, and grains in a vibrated silo. In all cases, the passage between consecutive particles was shown to exhibit a power law decay. In some conditions, anomalous statistics were evidenced as the exponent $\alpha$ of the power law decreased below two, and the first moment of the distribution could not be calculated. From this, a transition was identified from an unclogged state $(\alpha>2$, with well-defined averages) to a clogged state (where $\alpha \leqslant 2$ and the average flow rate tends to zero as the measuring time increases). After this definition, all the systems were encompassed in a tentative state diagram of clogging.

In this work we will investigate, for the case of sheep, aspects such as the flow rate through the door [26], the burst duration, the time lapse between two consecutive individuals, and the relationship among all these parameters. In addition, we will analyze the effect of some strategies that have already 
been successfully implemented in granular flows to enhance the flow properties. Presumably this will lead to improved knowledge that could eventually be extended to the case of people. Obviously humans and sheep are utterly different in many aspects, such as body constitution, navigation capabilities, communication skills, and intelligence. In addition, people and sheep usually behave in a very different way, because of politeness. But in some cases, for instance, in competitive situations, it is conceivable that people would shove one another selfishly. In those scenarios, the improvements we propose in Sec. IV may be worth checking, with the same methodology we put forward. Of course, this possibility remains to be tested.

In the next section we will explain how we carried out our observations, the methods we implemented, and the dataprocessing techniques. The third section will be devoted to the flow rates and the features of the probability distributions. Then we will provide results for the influence of the door size and the placement of an obstacle before the bottleneck. Finally, a summary of our conclusions is offered.

\section{EXPERIMENTAL PROCEDURES}

We have been granted access to a farm where a sheep herd is kept and to install there the necessary means to observe the animals in their everyday conditions. This has been supervised by two authors (L.M.F. and J.J.R.) who are veterinarian doctors. In order to collect a sizable amount of data and check for repeatability, we have restricted ourselves to the observation of a subset of the sheep flock: young mothers of the same age (so their body mass and size is very similar; the relevant dimension is the width at their hip, about $35 \mathrm{~cm}$ ). The sheep are Rasa Aragonesa breed, and those of the subset are separated from the flock and are kept in a barn. Every morning, they exit the barn and the door is closed. Feeders inside the barn are stocked with grain, and after some time the door $(77 \mathrm{~cm}$ wide) is open. As the sheep are eager to feed, they enter the barn in haste. We have installed several video cameras to register this entrance every day. Let us remark that this is the usual procedure in the farm, therefore this is just an observation of animal behavior in their regular routines. We have carried out this observation over several months. A new batch of sheep is drafted each month. These batches comprise between 60 and 110 sheep. Thus we have been able to check for repeatability between different days and groups of individuals. In addition, we have tested the validity of some approaches to ease clogging (as explained below) by comparing the data sets coming from different days of the same batch, with and without the improvements and the geometrical modifications considered.

The main objective of these observations is to obtain an accurate time log of the moment at which each sheep crosses the entrance. To do this, we have installed a zenithal camera inside the barn just above the door [Fig. 1(a)]. It is a standard video surveillance camera recording $704 \times 576$ pixels at 25 frames/sec. The timing is obtained by means of a procedure akin to the photo finish used in sport events. An image [such as the one shown in Fig. 1(c)] is built from the video frames in the following way. A line of pixels is sampled from every frame and stacked vertically; therefore, the horizontal dimension is the distance along the line, and the
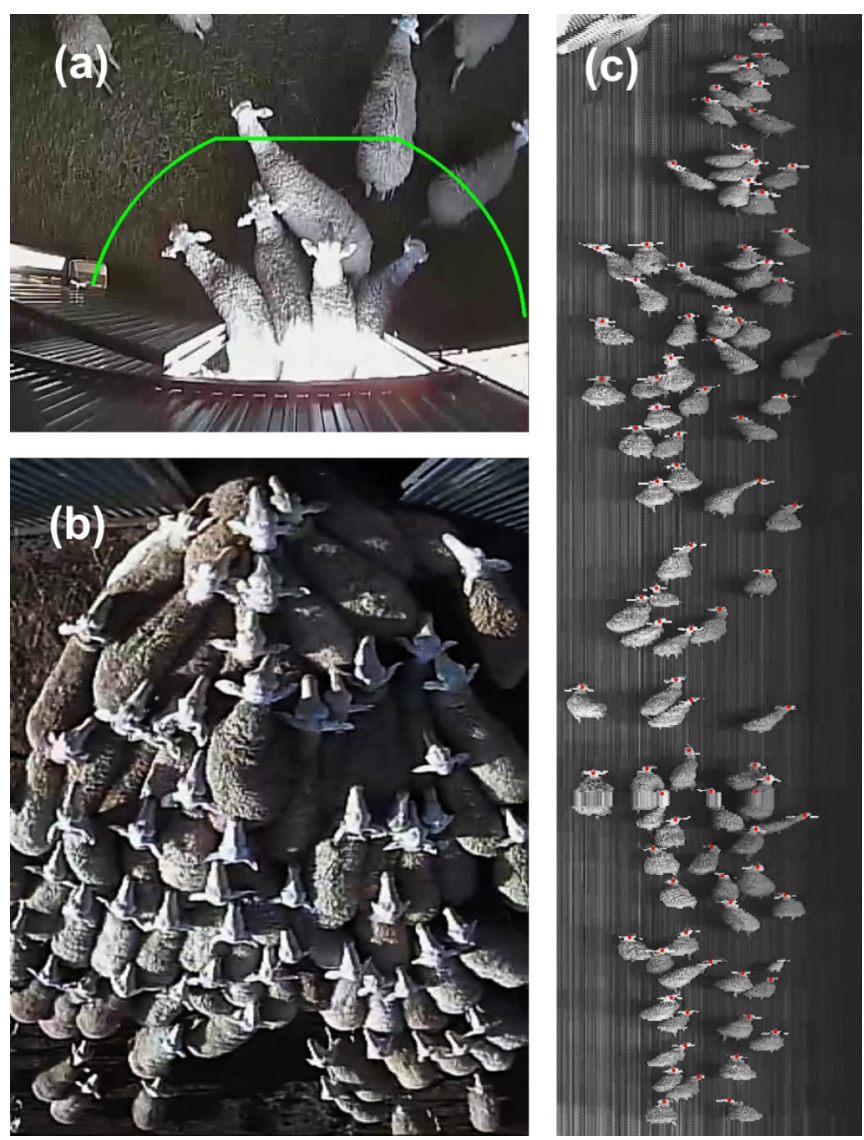

FIG. 1. (Color online) (a) A video frame obtained from the camera inside the barn when sheep are passing through a 77-cm-wide door. The green (light gray) line marks the pixels sampled to build the spatiotemporal diagram. (b) A photograph of the same entrance taken from above, at the outer side of the barn. (c) A spatiotemporal diagram of the sheep as they enter the barn. The head of each animal is marked with red (dark gray) dots in order to obtain the passage time.

vertical dimension is the time, in units of sampling period. In our case, we have taken a line at about a body length from the door [see Fig. 1(a)]. This is done because we are interested in counting animals that have crossed the door: as they can get stuck precisely at the door, it is reasonable to count them once they are at a body length from the door. The line is drawn with a segment parallel to the door line and two circular segments centered at the door side ends. We have checked that the outcomes are quite insensitive to this particular choice and to other details of the procedure. A subtlety we have introduced is to actually sample a thick line (five pixels wide), effectively multiplying by five the temporal scale of the image.

The second step is to mark every animal in the spatiotemporal diagram obtained as indicated [see Fig. 1(c)]. To this end, one has to choose a feature of the animal that can be identified in the photographs. After some trials, we have decided to mark the sheep head, as it is easily distinguishable. Again, we have made some tests adopting other rules, but the data are insensitive to the details of the method. The marks are done by hand, because we have found that the task of feature recognition is very difficult to automate in a reliable way. 
Once sheep are marked in the spatiotemporal diagrams, a simple image-processing program can produce a file with the passage time for every animal in the pack. The passage time information is processed to obtain the results presented in the following section. In Sec. IV, we will describe some modifications we have introduced in the entrance in order to improve the flow. In addition, we also installed cameras in another similar barn, where another pack of sheep of the same characteristics and size was fed, and recorded some entrances with the same method. We did this for control purposes, in order to be assured that our results do not depend on the specific features of a particular environment, and these data are not used in this paper.

\section{FLOW AND CLOGS}

With the data obtained as explained in the previous section, it is straightforward to plot the entrance time of each animal: in Fig. 2 these plots are displayed for 18 days of a particular pack, comprising 86 sheep. Near horizontal segments mark time lapses during which no individual crossed the door. A salient feature is the large variation from one day to another. This happens in all the packs and with all the entrance conditions we have implemented. A caveat is therefore pertinent, as several studies have focused almost exclusively on the evacuation time. With such a dispersion, it is difficult to describe the situation or to gauge the details of the evacuation process with just the average evacuation time. Moreover, one can ponder whether the evacuation time is the relevant piece of information. The answer, in our opinion, is in the negative. The fact that a given number of individuals (of the order of 100 in our case) can go through a door in $45 \mathrm{sec}$ instead of 40 , may be acceptable. Nevertheless, trouble may appear if a clog develops at the door, regardless of the total evacuation time. Casualties are often the result of asphyxiation due to a long clog, and from average evacuation times, clog durations cannot be straightforwardly calculated.

In order to quantify the magnitude of clogging events, we have resorted to the measurement of the time lapse $\Delta t$ between two consecutive individuals. We have recently argued [25] that the signature of a system prone to clogging is a power law tail in the probability distribution of the time lapses between elements. Indeed, if $P(\Delta t) \sim t^{-\alpha}$, with $\alpha$ smaller

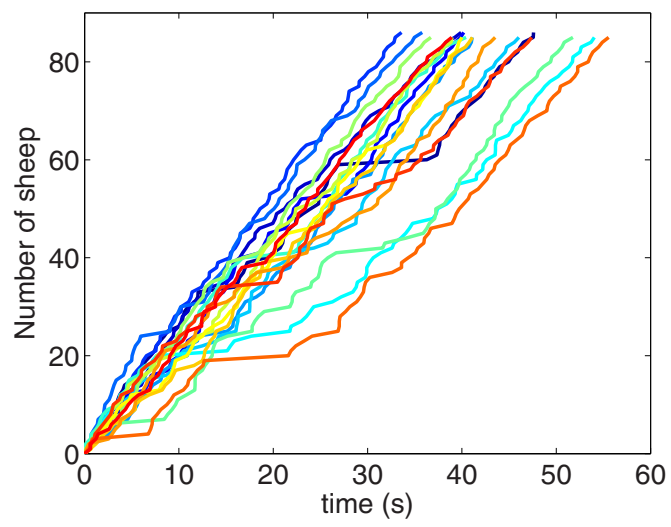

FIG. 2. (Color online) The number of sheep crossing the door as a function of time; each line corresponds to a different day. than two, then the average will not converge. This means that extreme events can occur, and that the mean is not a good descriptor of the system. More generally, in these situations, average quantities will probably be essentially determined by the largest event [27]. In our observations of sheep we have never measured an exponent $\alpha$ smaller than two, but in other conditions this could eventually happen.

A strict and rigorous data fit to obtain the exponent of a power law is not a straightforward procedure. It is not enough to visually establish a linear trend in a logarithmic-scaled histogram: instead, a more precise method has been put forward [28] that yields accurate results. It requires us to compute the complementary cumulative distribution, i.e., the probability that the time interval is greater or equal that a given value. The tail is then fitted to find $\alpha$, and the goodness of the fit is obtained (for instance, a $p$ value is given to assess the legitimacy of the result). The software described in Ref. [28] has been used for the power law fits given in this paper.

The statistics of the cumulated variable $T$ is revealing: a power law fits the tail nicely, giving in this case an exponent $\alpha=3.0$ (Fig. 3). This particular value of $\alpha$ indicates that the probability of finding long clogs decays rather quickly, so that the average converges, and therefore this is not an instance of the abovementioned anomalous statistics.

Let us now confront the problem of the definition of a clogging event. A power law in the probability distribution of $\Delta t$ means that there is not a typical parameter, viz., a characteristic scale to separate the time lapses between two animals into jammed and nonjammed situations. Then, in principle, the choice of the $\Delta t$ used to define clogging events (i.e., the time lapse between two consecutive animals above which the system is considered jammed, called here $\Delta t_{c}$ ) would be completely arbitrary. Nevertheless, some nuances

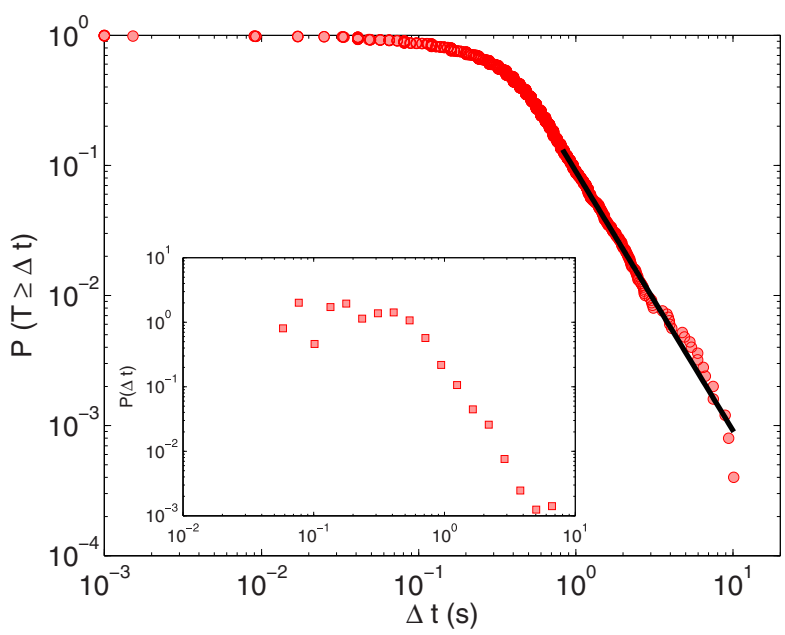

FIG. 3. (Color online) Power law tail of the time lapses $\Delta t$ : complementary cumulative distribution (also called survival function or reliability function) for the $77-\mathrm{cm}$-wide door. The fit of the power tail (black line) has been calculated with the method described in Ref. [28]. The particular values obtained for this fit are $\alpha=3.0$, $x_{\min }=0.82$. Inset: A normalized histogram representing the same data, with logarithmic bin widths. Note that increasing the number of bins will produce a noisier plot and the logarithmic scale in both graphs. 
must be discussed regarding this point. There is at least a typical time scale, which is given by the length of the animals $l$ divided by their velocity $v$, times the number of sheep that can go simultaneously through the door shoulder to shoulder (which is related to the ratio between door length and the animal width). A reasonable approximation for our case yields a time of about $0.5 \mathrm{sec}$. Time lapses much lower than this value are not actual clogs: they just occur naturally because of finite size effects. Another figure that can point to a good value for defining clogs is the place where the power law tail, as displayed in Fig. 3, begins in earnest. This number can be obtained from the algorithm used; we have consistently obtained values around 0.7 or $0.8 \mathrm{sec}$. Indeed, the straight line representing the fit in Fig. 3 is plotted from this value on. These two indicators suggest that a figure of the order of $1 \mathrm{sec}$ is a sound choice for $\Delta t_{c}$. Additional support for this will be given later.

Once $\Delta t_{c}$ is chosen, with the degree of arbitrariness explained, the passage of animals can be described as a continuous alternation between two regimes, "flowing" if $\Delta t<\Delta t_{c}$, and "interrupted" if $\Delta t \geqslant \Delta t_{c}$. We will describe the flowing regime by the burst size, which is the number of animals between two clogging events (or alternatively, a burst is a group of animals in which the largest time lapse is smaller than $\Delta t_{c}$ ). The interrupted regime is described by the duration of the clogging events, i.e., the distribution of the time lapses larger than $\Delta t_{c}$ (Fig. 3).

In Fig. 4(a) we represent the PDF of the burst size $S$ [29] divided by the mean burst size $\langle S\rangle$ obtained for $\Delta t_{c}=1 \mathrm{sec}$. It is evident that the distribution is exponential, a behavior that can be explained if there is a constant probability of clogging during the whole burst. In addition, the exponential distribution implies that a well-defined characteristic burst size can be obtained from the data. Hence the flowing regime features are well described, in a statistical sense (the moments of the distribution converge). The dangers of anomalous statistics are not present here. Let us note that burst duration also has an exponential distribution, a fact that should not come as a surprise, because as the average flow rate within the burst is well defined, as explained below, the burst can be indistinctly quantified in terms of size or duration.

We have previously stated that there is not a typical time scale in $\Delta t$ that allows us to select a neat and clear $\Delta t_{c}$ to split the flowing and interrupted regime. Therefore it is entirely admissible to take another value for $\Delta t_{c}$. We have found that for all the values explored the distribution of burst sizes $S$ is always exponential. The only change is the value of the mean burst size $\langle S\rangle$. As could be expected, $\langle S\rangle$ grows monotonously with $\Delta t_{c}$ for values above $0.8 \mathrm{sec}$ (a longer $\Delta t_{c}$ means that some bursts that where considered before as two different ones are now bound together). It is not possible to go much beyond $\Delta t_{c} \gtrsim 2$ due to the lack of statistics (the mean burst size grows so large that only a few burst are available to calculate the mean). These results are displayed in Fig. 4(b).

Finally, we can calculate the mean flow rate within the bursts. In order to do this, we take the burst size divided by the burst duration for all the bursts and calculate the weighted average (the weight being the number of animals in the burst). As the value of $\Delta t_{c}$ decreases, the length of the bursts becomes shorter because smaller time lapses are considered clogs. As
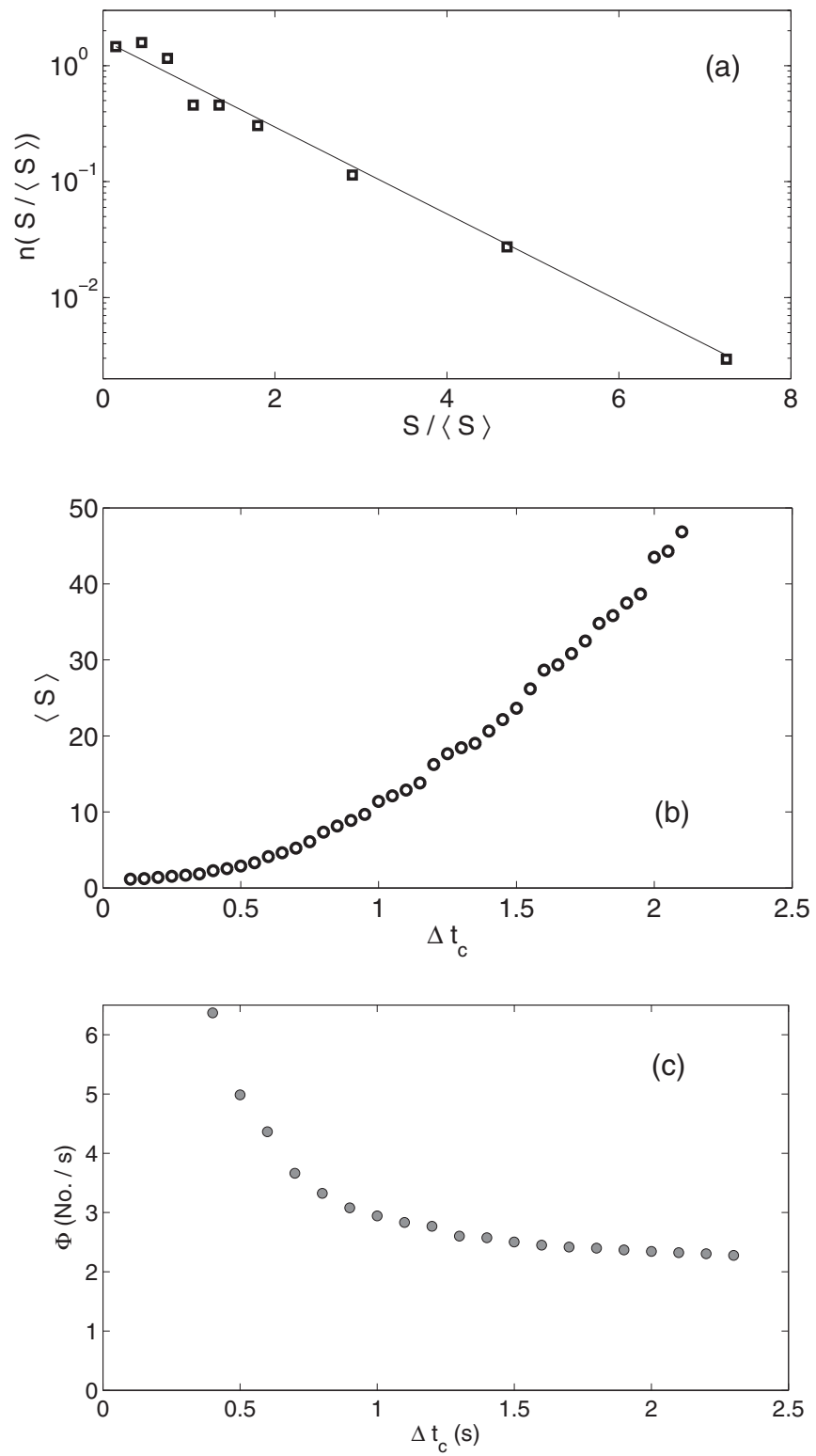

FIG. 4. (a) Histogram of the burst size normalized by the mean burst size. The line is a linear fit (note the semilogarithmic scale). For the definition of bursts, $\Delta t_{c}=1 s$ has been used. (b) The mean burst size $\langle S\rangle$ as a function of $\Delta t_{c}$. (c) The mean flow rate inside bursts as a function of $\Delta t_{c}$.

the number of animals is fixed (we always consider all the sheep), the mean flow rate necessarily increases: the same number of sheep are distributed in smaller, more compact bursts. From Fig. 4(c) it can be seen that below $\Delta t_{c} \approx 1 \mathrm{sec}$ the mean flow rate grows rapidly. This is still another indication of the limit for finite size effects. For $\Delta t_{c} \gtrsim 1 \mathrm{sec}$ the average flow within the bursts quickly approaches the value that is obtained when dividing the total number of sheep by the mean evacuation time, which is about two sheep per second.

\section{ENHANCEMENTS}

Let us now consider whether some strategies that have been successfully used to prevent or reduce the clogging probability 
in inert granular media are also beneficial for active matter. Two checks have been performed: a change in the door size, and the placement of an obstacle before the door. Both will be analyzed in the light of the results presented in Sec. III.

The first modification we will discuss is the change of the entrance width (see Ref. [26] for an analysis of pedestrian flow when changing the door size). As explained before, the exponent $\alpha=3.0$ obtained for a 77-cm-wide door implies that the average converges. In order to attain a situation with $\alpha<2$, the door size should be decreased, but this is unfeasible for ethical reasons (it could cause injuries to the animals). What we have done instead is to widen the door and see if the inverse effect is observed, i.e., if $\alpha$ grows larger. If this is so, then it is expected that a narrower door would make $\alpha$ decrease, eventually reaching a clogged state as defined in Ref. [25]. We therefore changed the door to a similar one but $94 \mathrm{~cm}$ wide (in the following, this door will be referred to as the large door, while the preceding one, $77 \mathrm{~cm}$ wide, will be called the small door). We implemented the procedure explained above and calculated the burst size and the lapse time probability distribution function.

The second modification introduced is the placement of an obstacle (as reported, for instance, in Ref. [30]) before the door. This obstacle is a cylinder with a diameter of $114 \mathrm{~cm}$ and higher than the sheep, so it hides the door from the animals. It is a section of a concrete drainpipe, and it was placed in such a way that its nearest point was at $80 \mathrm{~cm}$ from the door, and centered in the middle of the opening. The aim of this test was to check whether this arrangement will reduce the clogging probability. An experiment with inert granular media [31] demonstrated how this strategy could reduce clogging by a factor of more than one hundred. In the case of animals, it is not feasible to collect such a large amount of data as in the laboratory, so only the analysis of the burst size and the PDF of $\Delta t$ is available. Again, experiments were carried out following the procedure explained previously. We took care to do this with several packs of sheep, and for each pack we recorded the entrance without obstacle for about 2 weeks, then with the obstacle for another 2 weeks. In this way, we checked that the behavior of the animals was similar and we were confident to aggregate all the data.

The analysis of $\Delta t$ reveals power law tails in all the situations investigated (small and large door, with and without obstacle), as shown in Fig. 5 (see also Table I). In the case of the larger door, a higher exponent $\alpha=3.4$ is found, meaning that the system is less prone to clogging. One can therefore make the educated guess that a smaller door would lead to a distribution with a lower $\alpha$, eventually reaching the threshold $\alpha<2$. The precise door size corresponding to this threshold can not be estimated, because it would involve an extrapolation and $\alpha$ could depend on the door size in a nonlinear fashion (some indications come from experiments in granular flows through an orifice [21]).

The placement of the obstacle leads to an increase of the exponent in the distribution of $\Delta t$ : from 3.4 to 4.8 for the large door, and from 3.0 to 4.0 in the case of the small door. It is therefore quite natural to assume that an obstacle lessens the tendency of the system to clog. Of course, we have explored a system only in a state where $\alpha>2$, but it is not risky to assume that this enhancement will also work for $\alpha<2$ (a clogged

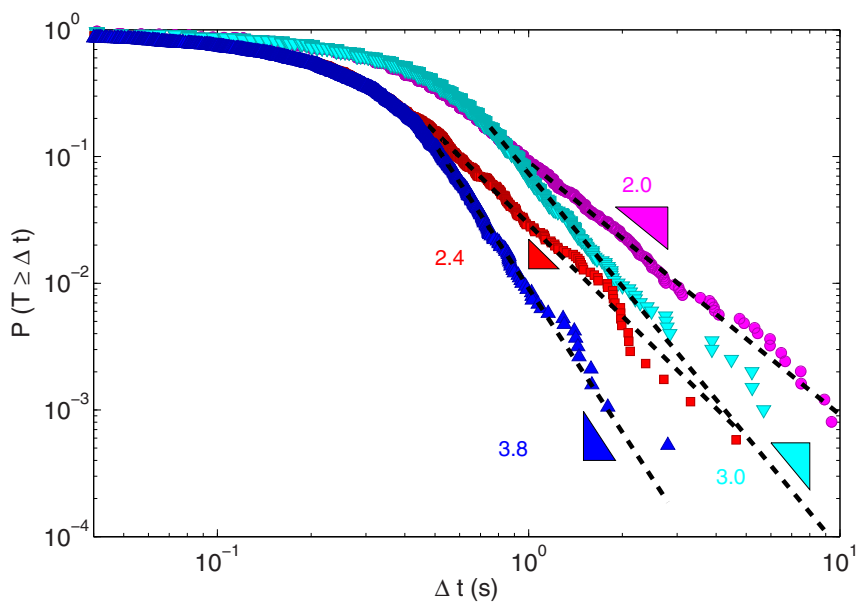

FIG. 5. (Color online) Complementary cumulative distributions of $\Delta t$ corresponding to the following cases: small door, no obstacle (pink o); small door, with obstacle (cyan $\nabla$ )); large door, no obstacle (red $\square$ ); large door, with obstacle (blue $\triangle$ ). Note that the slopes indicated in the graphs differ from $\alpha$ in 1 , as they correspond to cumulative distributions.

state [25]). Let us also remark that we have only performed this experiment with the obstacle at a fixed position. In order to find the optimal reduction of the clogging probability, the distance to the door is crucial [32-34]. This exploration is costly in terms of the time needed and we have not attempted it.

The burst size distribution remains exponential when an obstacle is present and the door is enlarged. The only change is the value of the characteristic parameter of the exponential, which of course can be related to $\langle S\rangle$. In Fig. 6(a) we reproduce the same data as in Fig. 4 (the values of $\langle S\rangle$ versus $\Delta t_{c}$ for the four scenarios investigated: with and without an obstacle, for large and small doors). It provides evidence that for both door sizes the obstacle is beneficial in terms of the burst sizes, because $\langle S\rangle$ is bigger in this case, regardless of the particular value chosen for $\Delta t_{c}$. In addition, the larger the door the bigger $\langle S\rangle$. Note that we cannot calculate $\langle S\rangle$ for arbitrarily long $\Delta t_{c}$ because bursts become extremely long and eventually take the whole lot of individuals recorded.

In Fig. 6(b) we plot the ratio $r$ of the mean burst sizes with and without obstacle both for the small and the large doors. Note that below a certain $\Delta t_{c}$ the mean burst size is almost unaffected by presence of the obstacle (alternatively, $r \approx 1$ ). For the large door this value is $\Delta t_{c} \approx 0.4$ and for the small door $\Delta t_{c} \approx 0.8$. These numbers are to be compared with the

TABLE I. Values of some parameters for the different enhancements: $\alpha$ is the exponent of the power law tail, $t_{\min }$ is the value of $\Delta t$ above which the fit is valid, $N$ is the total number of animals recorded, and $t_{1}$ is the average evacuation time per animal.

\begin{tabular}{lccccc}
\hline \hline Door & Obstacle & $\alpha$ & $t_{\min }(\mathrm{s})$ & $N$ & $t_{1}(\mathrm{~s})$ \\
\hline Small $(77 \mathrm{~cm})$ & No & 3.0 & 0.82 & 2535 & 0.50 \\
Small $(77 \mathrm{~cm})$ & Yes & 4.0 & 0.75 & 2025 & 0.47 \\
Large $(94 \mathrm{~cm})$ & No & 3.4 & 0.48 & 1742 & 0.30 \\
Large $(94 \mathrm{~cm})$ & Yes & 4.8 & 0.51 & 1922 & 0.27 \\
\hline \hline
\end{tabular}



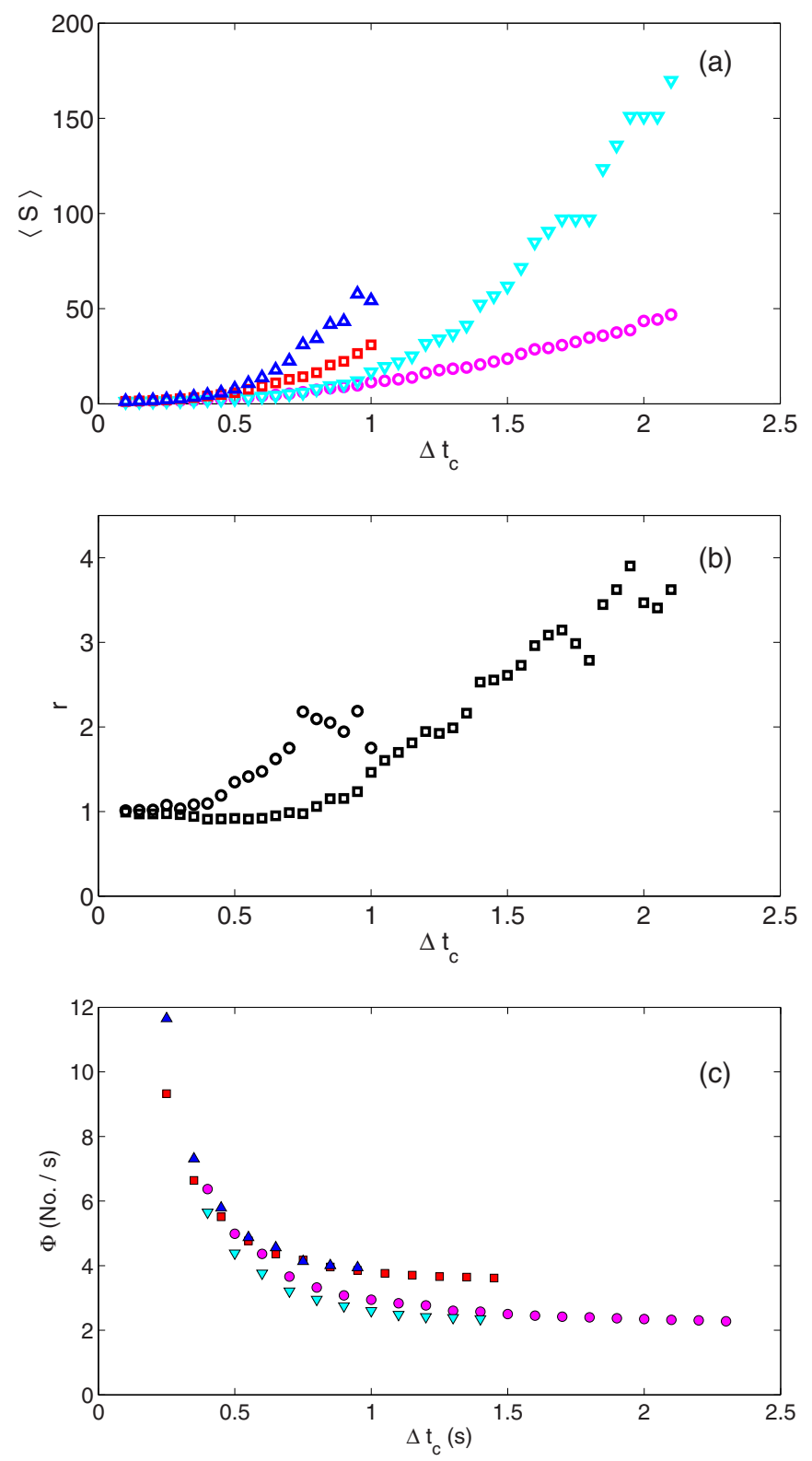

FIG. 6. (Color online) (a) Mean burst size $\langle S\rangle$ as a function of the value $\Delta t_{c}$ chosen for the definition of clogs. The symbols are the same that in Fig. 5: small door, no obstacle (pink o); small door, with obstacle (cyan $\nabla$ ); large door, no obstacle (red $\square$ ); large door, with obstacle (blue $\triangle$ ) (b) Ratio $r$ of mean burst size with and without obstacle as a function of $\Delta t_{c}$ for the large door (o) and the small door( $\square$ ). (c) The mean flow rate within bursts as a function of $\Delta t_{c}$ [symbols correspond to the same cases as explained in (a)].

values of $t_{\min }$ provided in Table I. This is yet another indication of the finite size effects limit, which can be used as a threshold for the definition of clogs. Above this value, indeed, if one decides to take a more stringent value of $\Delta t_{c}$, i.e., a larger one, meaning that only long clogs will be considered, then the burst size will increase, so the improvement gained by placing an obstacle would be still bigger.

Finally, let us consider the mean flow rate inside bursts [as was done in Fig. 4(c)] for both door sizes, with and without obstacles. These are displayed in In Fig. 6(c). As explained above, the limit for large $\Delta t_{c}$ must tend to the mean flow rate (the inverse of $t_{1}$ in Table I). Note that even though the obstacle changes the likelihood of long clogs, its presence does not change the mean flow rate within bursts noticeably.

\section{CONCLUSIONS}

We have observed the behavior of a sheep herd when going through a door in a competitive fashion. From the recorded passage time of the animals as they cross the door, we have studied basically the burst size (groups of animals passing uninterruptedly between clogs) and the distribution of time lapses between consecutive animals. As the burst sizes are exponentially distributed, the mean burst size can be easily obtained, thus providing a characteristic parameter that correctly describes the flowing regime. On the other hand, the distribution of lapse times displays a power law tail. Care must be taken when providing averages if the exponent of the power law is smaller than two, because in this case the first moment of the distribution would not converge and the mean will be dominated essentially by the largest event. This condition has been avoided in the present work, but if it were the case, the possibility of extreme events (very long clogs) should be carefully considered. Therefore, emphasis on the duration of clogging events instead of averages such as evacuation time is pertinent.

We have checked that some procedures that reduce the clogging probability in the case of inert granular media can be successfully applied to active matter, at least in principle. The obvious action of widening the orifice has a beneficial effect, as well as placing an obstacle before the door. The effectiveness of these approaches can be gauged by measuring the exponent of the power law tail in the time lapse distribution.

It is fitting to point out that this work may lead to a better understanding of how to avoid the dangers of clogging in people crowds going through emergency exits or narrow passages. The relevance that this could have in architectural design is of course noteworthy. The choice of sheep is quite sound as an intermediate step between inert grains and humans, and the scope of the present analysis can eventually be broadened and help to better manage human throngs. This is, however, something that remains to be analyzed and checked. Whether the placement of an obstacle is beneficial for a group of people shoving one another will be difficult to check experimentally, but perhaps computer simulations could shed light on this issue.

\section{ACKNOWLEDGMENTS}

We are indebted to Luis Fernado Urrea for technical help and Tomás Yagüe, at whose farm we recorded the films. Financial support from PIUNA (Universidad de Navarra), Project FIS2011-26675 from Ministerio de Economía y Competitividad (Spanish Government) and Mutua Montañesa is gratefully acknowledged. We also benefited from discussions with D. Maza, R. Cruz Hidalgo, C. Lozano, E. Altshuler, and C. del Valle. 
[1] D. Helbing and P. Molnár, Social force model for pedestrian dynamics, Phys. Rev. E 51, 4282 (1995).

[2] A. Schadschneider, W. Klingsch, H. Klüpfel, T. Kretz, C. Rogsch, and A. Seyfried, Evacuation dynamics: Empirical results, modeling and applications, in Encyclopedia of Complexity and System Science, edited by B. Meyer (Springer, Berlin, 2008), pp. 3142-3176.

[3] D. Helbing, A. Johansson, and H. Z. Al-Abideen, Dynamics of crowd disasters: An empirical study, Phys. Rev. E 75, 046109 (2007).

[4] M. Boltes and A. Seyfried, Collecting pedestrian trajectories, Neurocomputing 100, 127 (2013).

[5] D. Yanagisawa, A. Kimura, A. Tomoeda, R. Nishi, Y. Suma, K. Ohtsuka, and K. Nishinari, Introduction of frictional and turning function for pedestrian outflow with an obstacle, Phys. Rev. E 80, 036110 (2009).

[6] M. Moussaï, D. Helbing, and G. Theraulaz, How simple rules determine pedestrian behavior and crowd disasters, Pub. Natl. Acad. Sci. USA 108, 6884 (2011).

[7] F. Alonso-Marroquín, J. Busch, C. Chiew, C. Lozano, and A. Ramírez-Gómez, Simulation of counterflow pedestrian dynamics using spheropolygons, Phys. Rev. E 90, 063305 (2014).

[8] T. Ezaki, D. Yanagisawa, and K. Nishinari, Pedestrian flow through multiple bottlenecks, Phys. Rev. E 86, 026118 (2012).

[9] S. Bouzat and M. N. Kuperman, Game theory in models of pedestrian room evacuation, Phys. Rev. E 89, 032806 (2014).

[10] T. Vicsek and A. Zafeiris, Collective motion, Phys. Rep. 517, 71 (2012).

[11] J. Gachelin, G. Miño, H. Berthet, A. Lindner, A. Rousselet, and E. Clément, Non-newtonian viscosity of Escherichia coli suspensions, Phys. Rev. Lett. 110, 268103 (2013).

[12] M. K. Gdoura, R. Löhner, E. Haug, and B. Gawenat, On the influence of columns in densely populated corridors, in Transportation Research Procedia, Vol. 2, edited by W. Daamen, D. C. Duives, and S. P. Hoogendoorn, Proceedings of the Conference on Pedestrian and Evacuation Dynamics, 22-24 October 2014, Delft, the Netherlands (Elsevier, Amsterdam, 2014), pp. 2-9.

[13] C. Saloma, G. J. Perez, G. Tapang, M. Lim, and C. PalmesSaloma, Self-organized queuing and scale-free behavior in real escape panic, Pub. Natl. Acad. Sci. USA 100, 11947 (2003).

[14] E. Altshuler, O. Ramos, Y. Núñez, J. Fernández, A. J. BatistaLeyva, and C. Noda, Symmetry breaking in escaping ants, Am. Nat. 166, 643 (2005).

[15] S. Boari, R. Josens, and D. R. Parisi, Efficient egress of escaping ants stressed with temperature, Plos ONE 8, e81082 (2013).

[16] A. Sobhani, M. Sarvi, D. Duives, O. Ejtemani, K. Aghabayk, and S. Hoogendoorn, Exploring the relationship of exit flow and jam density in panic scenarios using animal dynamics, in Transportation Research Procedia, Vol. 2, edited by W. Daamen, D. C. Duives, and S. P. Hoogendoorn, Proceedings of the Conference on Pedestrian and Evacuation Dynamics, 22-24 October 2014, Delft, the Netherlands (Elsevier, Amsterdam, 2014), pp. 745-751.
[17] A. John, A. Schadschneider, D. Chowdhury, and K. Nishinari, Trafficlike collective movement of ants on trails: Absence of a jammed phase, Phys. Rev. Lett. 102, 108001 (2009).

[18] N. Shiwakoti, M. Sarvi, G. Rose, and M. Burd, Enhancing the safety of pedestrians during emergency egress, Transport. Res. Rec. 2137, 31 (2009).

[19] J. S. Tubbs and B. J. Meacham, Egress Design Solutions (John Wiley \& Sons, Hoboken, NJ, 2007).

[20] K. To, P.-Y. Lai, and H. K. Pak, Jamming of granular flow in a two-dimensional hopper, Phys. Rev. Lett. 86, 71 (2001).

[21] I. Zuriguel, A. Garcimartín, D. Maza, L. A. Pugnaloni, and J. M. Pastor, Jamming during the discharge of granular matter from a silo, Phys. Rev. E 71, 051303 (2005).

[22] S. Tewari, M. Dichter, and B. Chakraborty, Signatures of incipient jamming in collisional hopper flows, Soft Matter 9, 5016 (2013).

[23] J. Tang and R. P. Behringer, How granular materials jam in a hopper, Chaos 21, 041107 (2011).

[24] I. Zuriguel, L. A. Pugnaloni, A. Garcimartín, and D. Maza, Jamming during the discharge of grains from a silo described as a percolating transition, Phys. Rev. E 68, 030301(R) (2003).

[25] I. Zuriguel, D. R. Parisi, R. C. Hidalgo, C. Lozano, A. Janda, P. A. Gago, J. P. Peralta, L. M. Ferrer, L. A. Pugnaloni, E. Clément, D. Maza, I. Pagonabarraga, and A. Garcimartín, Clogging transition of many-particle systems flowing through bottlenecks, Sci. Rep. 4, 7324 (2014).

[26] A. Seyfried, O. Passon, B. Steffen, M. Boltes, T. Rupprecht, and W. Klingsch, New Insights into Pedestrian Flow Through Bottlenecks, Transport. Sci. 43, 395 (2009).

[27] D. Sornette, Critical Phenomena in Natural Sciences (Springer, Heidelberg, 2000).

[28] A. Clauset, C. R. Shalizi, and M. E. J. Newman, Power-law distributions in empirical data, SIAM Rev. 51, 661 (2009).

[29] For the computation of bursts, we linked the last animal of each day with the first one of the following day.

[30] D. Helbing, L. Buzna, A. Johansson, and T. Werner, SelfOrganized Pedestrian Crowd Dynamics: Experiments, Simulations, and Design Solutions, Transport. Sci. 39, 1 (2005).

[31] I. Zuriguel, A. Janda, A. Garcimartín, C. Lozano, R. Arévalo, and D. Maza, Silo clogging reduction by the presence of an obstacle, Phys. Rev. Lett. 107, 278001 (2011).

[32] C. Lozano, A. Janda, A. Garcimartín, D. Maza, and I. Zuriguel, Flow and clogging in a silo with an obstacle above the orifice, Phys. Rev. E 86, 031306 (2012).

[33] A. Kirchner, K. Nishinari, and A. Schadschneider, Friction effects and clogging in a cellular automaton model for pedestrian dynamics, Phys. Rev. E 67, 056122 (2003).

[34] T. Matsuoka, A. Tomoeda, M. Iwamoto, K. Suzuno, and D. Ueyama, Effects of an obstacle position for pedestrian evacuation: SF model approach, in Traffic and Granular Flow '13, edited by M. Chraibi, M. Boltes, A. Schadschneider, and A. Seyfried (Springer, Heidelberg, 2015), pp. 163-170. 